\title{
Saint-Martin de Blanquefort (Gironde) : apport d'une fouille de faible ampleur à la connaissance d'un cimetière paroissial
}

Juliette Masson, Hélène Réveillas et Mikaël Rouzic

\author{
(2) OpenEdition \\ Journals \\ Édition électronique \\ URL : https://journals.openedition.org/cem/14772 \\ DOI : 10.4000/cem. 14772 \\ ISSN : 1954-3093 \\ Éditeur \\ Centre d'études médiévales Saint-Germain d'Auxerre
}

Référence électronique

Juliette Masson, Hélène Réveillas et Mikaël Rouzic, « Saint-Martin de Blanquefort (Gironde) : apport d'une fouille de faible ampleur à la connaissance d'un cimetière paroissial », Bulletin du centre d'études médiévales d'Auxerre | BUCEMA [En ligne], 21.2 | 2017, mis en ligne le 07 février 2018, consulté le 22 septembre 2022. URL : http://journals.openedition.org/cem/14772 ; DOI : https://doi.org/10.4000/ cem. 14772

Ce document a été généré automatiquement le 22 septembre 2022.

\section{(c) (P)}

Creative Commons - Attribution - Pas d'Utilisation Commerciale - Partage dans les Mêmes Conditions 4.0 International - CC BY-NC-SA 4.0

https://creativecommons.org/licenses/by-nc-sa/4.0/ 
Saint-Martin de Blanquefort (Gironde) : apport d'une fouille de faible ampleur à la connaissance d'un cimetière paroissial

Juliette Masson, Hélène Réveillas et Mikaël Rouzic

\section{NOTE DE L'AUTEUR}

Avec la collaboration de Valérie Marache, céramologue gestionnaire du mobilier, Centre d'archéologie préventive de Bordeaux métropole. 
1 Blanquefort est une des vingt-huit communes du territoire métropolitain de Bordeaux (Gironde). De nombreux indices d'une occupation dans ce secteur, dès l'Antiquité, sont mentionnés dans la Carte Archéologique de la Gaule relative à la Gironde $^{1}$. Située au nord de Bordeaux, cette commune est connue localement pour le château de Blanquefort, dont les vestiges les plus anciens sont attribuables

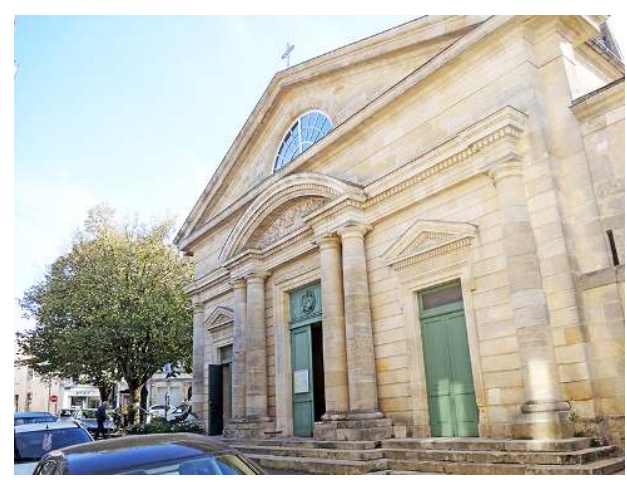
$\mathrm{au} \mathrm{XI}^{\mathrm{e}}$ siècle. Dès le $\mathrm{XIX}^{\mathrm{e}}$ siècle, des fragments de tegulae utilisés en remploi sont remarqués dans les murs du château et des monnaies romaines ainsi que de la céramique protohistorique sont mises au jour à l'occasion d'une fouille sur le site castral.

2 Un diagnostic a été réalisé par l'Inrap en 2012, dans le cadre du réaménagement de la place de l'église paroissiale Saint-Martin de Blanquefort ${ }^{2}$. Il a révélé une occupation funéraire illustrée par une inhumation en pleine terre avec une logette céphalique, des sépultures sans architecture en pierre, mais ayant pu avoir un couvercle en matériau périssable, des sépultures aménagées dans un coffrage en pierre avec logette céphalique et des sarcophages trapézoïdaux. Il est mentionné également une construction rectangulaire à quatre compartiments, réalisée dans l'enceinte du cimetière, et qui pourrait être un caveau ou un pourrissoir.

3 Ces résultats ont amené à la prescription d'une fouille réalisée à l'automne 2014. Le projet consistait en la mise en place d'une fontaine nécessitant l'aménagement d'une cuve et de deux chambres techniques. Placée à une dizaine de mètres au sud de l'église, la surface affectée était réduite : l'emprise de la fouille représentait seulement $9 \mathrm{~m}^{2}$, pour une profondeur maximum de $1,30 \mathrm{~m}$.

\section{État des connaissances}

\section{Une église romane en grande partie disparue}

4 L'église paroissiale Saint-Martin de Blanquefort, orientée nord-ouest - sud-est, résulte du profond remaniement d'un édifice médiéval : seule une abside romane subsiste pour les anciennes parties orientales. D’après les dernières études menées par les associations historiques locales, l'église des $\mathrm{XI}^{\mathrm{e}}-\mathrm{XII}^{\mathrm{e}}$ siècles aurait été détruite par un incendie et reconstruite dans le style gothique aux $\mathrm{Xv}^{\mathrm{e}}$ et $\mathrm{xvI}^{\mathrm{e}}$ siècles, avant une restauration complète dans un style néoclassique au cours du XIX siècle (fig. 1 et 2). 
Fig. 1 - Localisation de l'église paroissiale Saint-Martin de Blanquefort et signalisation de la zone fouillée en 2014

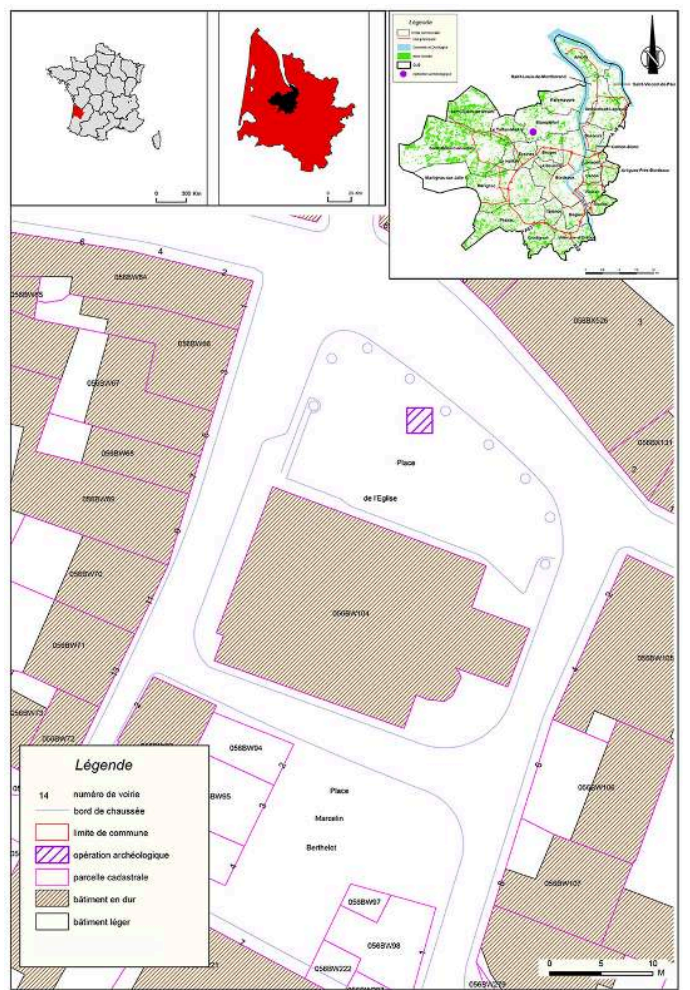

DAO Bordeaux Métropole

Fig. 2 - Saint-Martin de Blanquefort : élévation nord, façade de l'église paroissiale, reconstruite au $\mathrm{XIX}$ siècle dans le style néoclassique

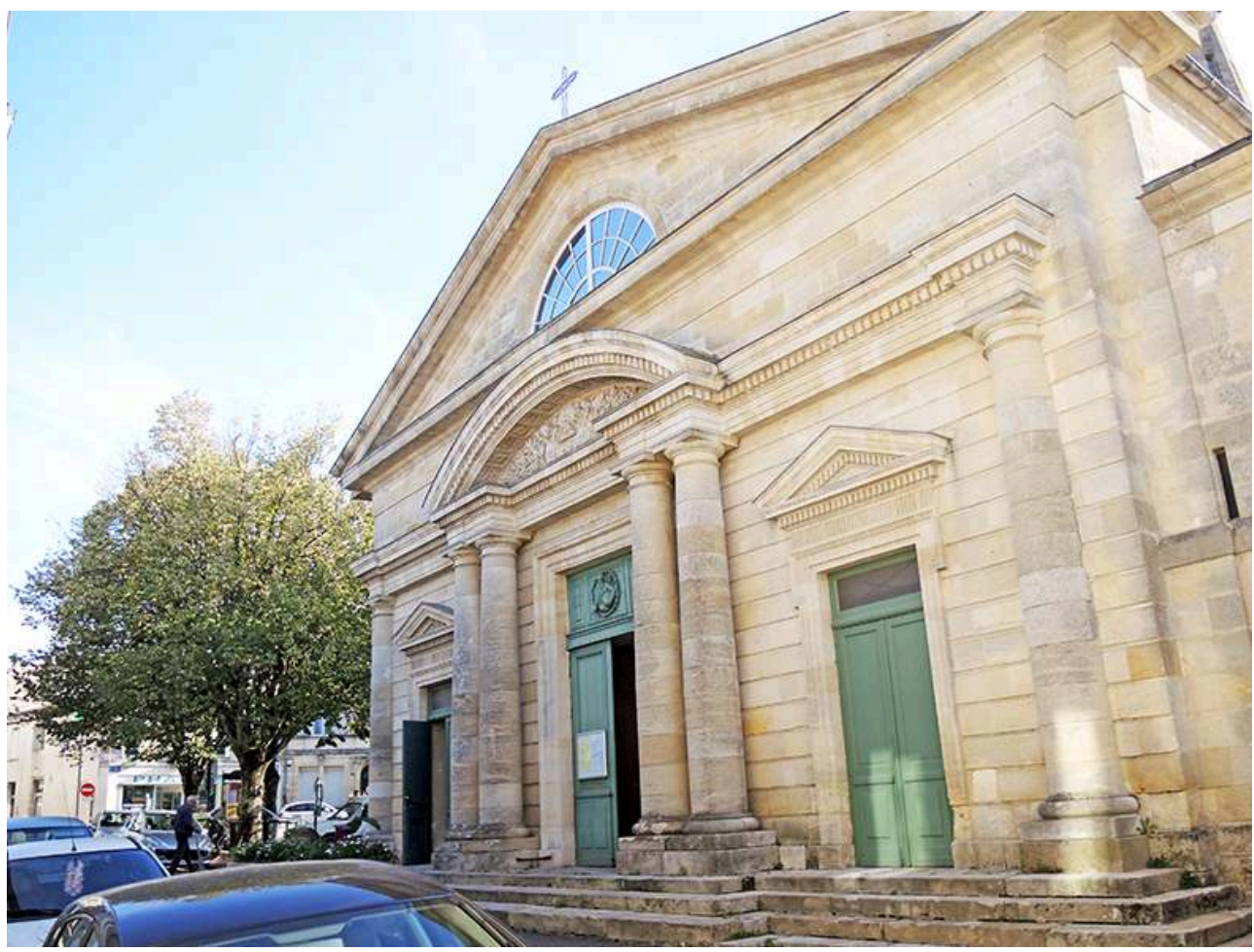

Cl. J. Masson 
La plus ancienne mention de la paroisse, alors située en milieu rural, date du XII ${ }^{\mathrm{e}}$ siècle. Elle est en effet signalée en 1165 dans le cartulaire de Sainte-Croix parmi les possessions de l'abbaye suburbaine.

6 Un ancien plan de l'église, non daté, permet d'observer un état disparu de l'édifice (fig. 3).

Fig. 3 - Saint-Martin de Blanquefort : plan ancien de l'église et du cimetière

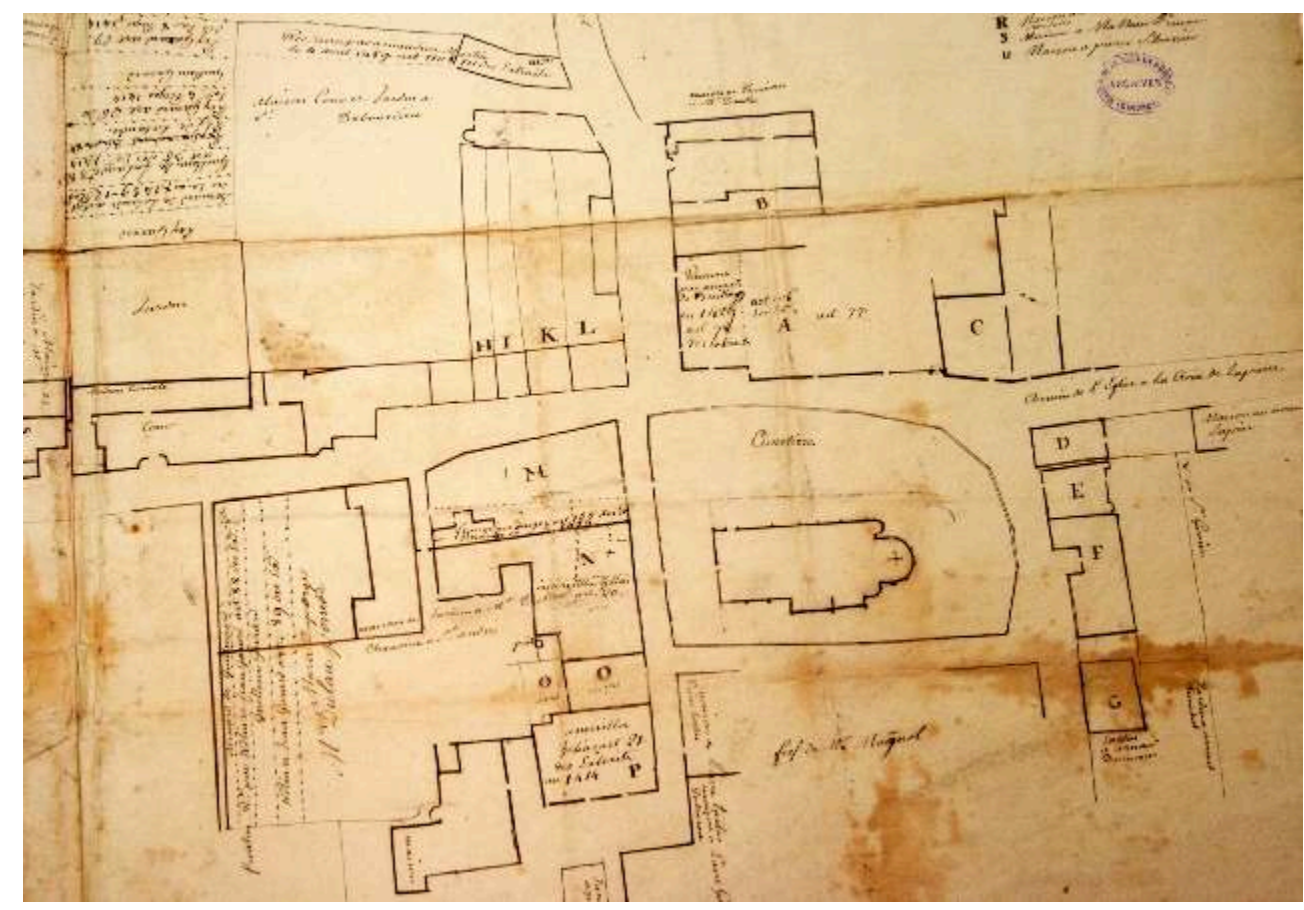

Plan fourni par M. Henri Bret (Cercle historique de Blanquefort), Archives de la ville de Bordeaux. Cote et date non communiquées.

7 On peut distinguer la profonde abside du chevet cantonnée de deux absidioles. La nef paraît très large, aussi large que les parties orientales, et des contreforts semblent rythmer l'élévation extérieure de l'édifice, sauf la façade occidentale. Deux annexes, ou chapelles, flanquent la nef au sud. Une porte ouvre à l'ouest et une autre ouverture est ménagée au nord dans la travée occidentale de la nef, amenant vers le cimetière. Ce dernier est signalé autour de l'église par un trait symbolisant une clôture, où des ouvertures sont figurées. Cette clôture accuse un angle au sud-ouest, car elle suit le tracé de la voirie, contrairement au nord et à l'est, où elle souligne un espace aux contours arrondis ou à pans coupés, donnant ainsi l'impression que cet espace était plus grand et fut tronqué par l'aménagement des rues. L'église apparaît dans la moitié sud de la zone ainsi délimitée, mais elle a pu être plus centrale auparavant. L'accès au cimetière est signalé à trois endroits avec deux ouvertures à l'ouest, dont l'une est face à la porte de l'église, et une autre au nord.

8 Les textes renseignent peu sur l'église ancienne, mais quelques indices peuvent toutefois être glanés au gré des comptes rendus de visite. Un texte daté de 1734 décrit une église bâtie de pierres, de chaux et de sable. La longueur donnée est de 60 pieds, pour une largeur de 46 pieds et 27 pieds de hauteur. L'église est plafonnée et carrelée, « les murailles en sont bonnes, blanchies au-dedans et non recrépies par dehors, les fenêtres sont vitrées garnies de barres de fer avec des châssis de fil d'archal ; les portes 
sont en bon état et ferment à clef ». Est encore mentionné " un clocher en forme de tour carrée qui est au-dessus de la chapelle saint George couvert d'ardoises, les murs en sont bons, l'escalier en bon état et fermé à clef, il y a deux cloches ». Le même texte évoque le cimetière, qui « environne l'église, est entouré de murailles, ne ferme point à clef, assez fermé d'ailleurs, il n'y a point de grillages aux entrées, il y a une grande croix de pierre au milieu, il n'y a point d'endroit séparé pour les enfants baptisés, on ne porte point les enfants décédés sans baptême, on a soin de faucher l'herbe qui vient dans le cimetière, il n'y a point de marché ni chemin public $»^{3}$.

Le clocher mentionné dans cette visite ne se détecte pas sur le plan précédent (cf. fig. 3), mais peut-être est-ce celui qui apparaît sur le cadastre de 1843 (fig. 4), établi à une date où une église de style néoclassique a remplacé la précédente. Étant mentionné au-dessus d'une chapelle et sans croisée visible sur le plan ancien, ce clocher pourrait être lié à d'hypothétiques aménagements gothiques des $\mathrm{Xv}^{\mathrm{e}}-\mathrm{XVI}^{\mathrm{e}}$ siècles, mais il indique probablement que la reconstruction néoclassique est antérieure à 1734 .

Fig. 4 - Extrait du cadastre daté de 1843

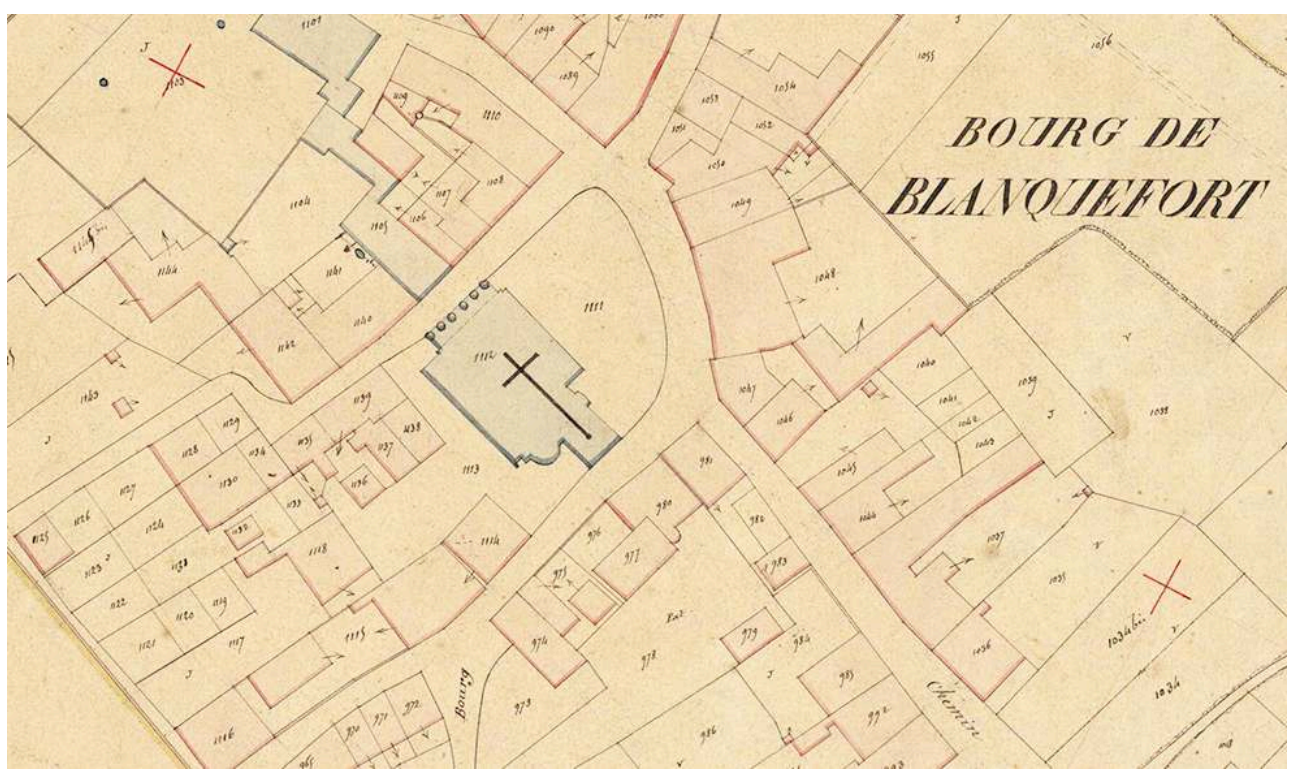

On distingue l'absidiole d'origine romane conservée au sud de l'édifice néoclassique. Archives départementales de la Gironde, cote 3 P 056/063.

\section{Des indices d'une occupation funéraire pour le haut Moyen Âge}

Une fouille réalisée dans l'église en 1984 par B. Fayolle-Lussac a mis au jour deux sarcophages mérovingiens, dont un est entreposé dans l'actuel cimetière de la commune (fig. 5) . 
Fig. 5 - Sarcophage découvert dans l'église Saint-Martin, conservé dans le cimetière actuel de Blanquefort

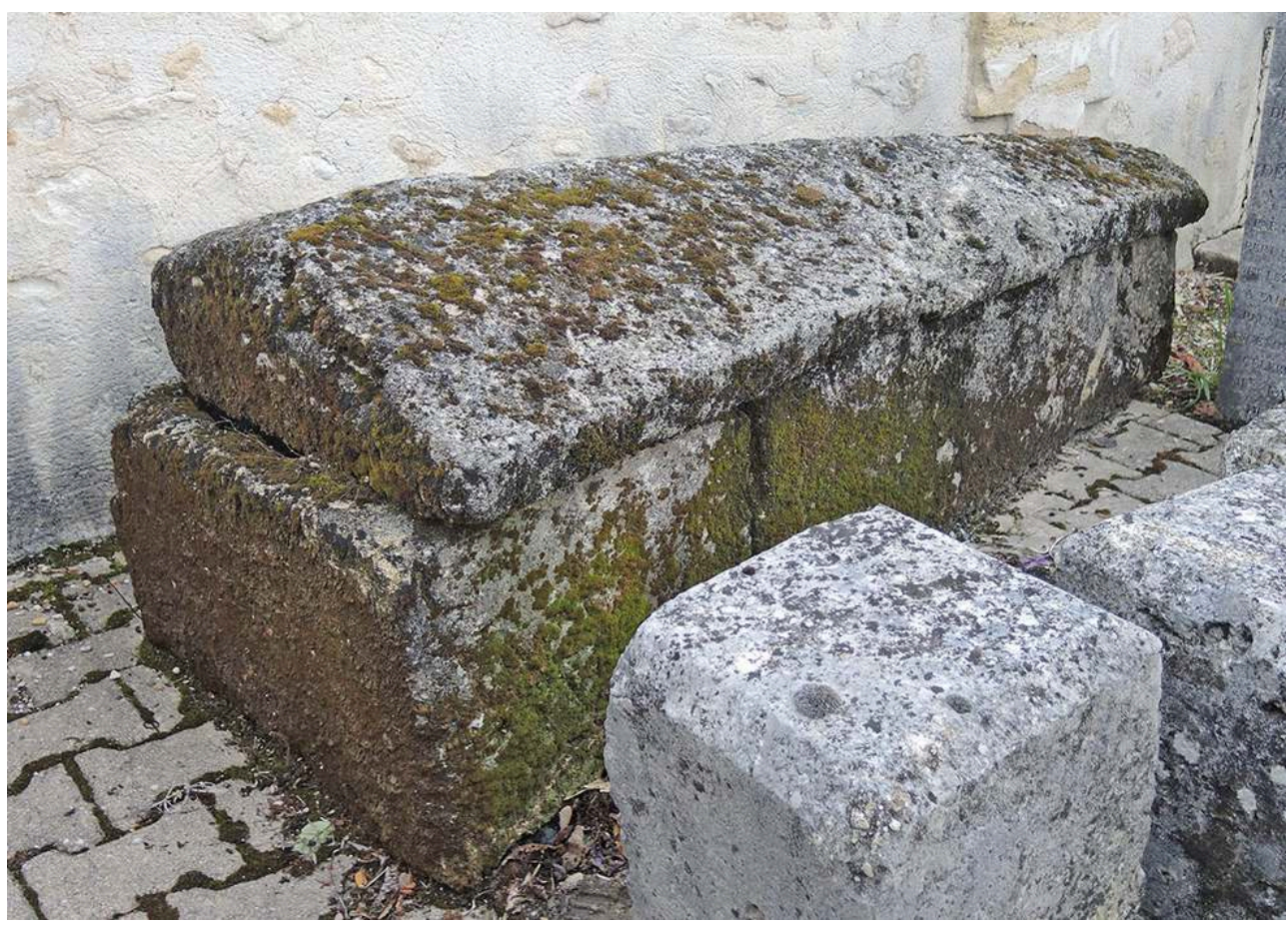

Cl. J. Masson

11 Ce sarcophage, indice d'une occupation du site pour la période mérovingienne, comporte une cuve longue de $1,97 \mathrm{~m}$ et large de $0,68 \mathrm{~m}$ à la tête et de $0,17 \mathrm{~m}$ au pied mesures prises en partie haute - pour une profondeur de 0,37 $\mathrm{m}$ et des parois épaisses de 6 à $8 \mathrm{~cm}$. Le couvercle, en bâtière et sans décor ni strie visible, est long de $1,98 \mathrm{~m}$, large de $0,64 \mathrm{~m}$ côté tête et de $0,33 \mathrm{~m}$ côté pieds, pour une épaisseur de 0,13-0,23 m comprenant un chanfrein de $6 \mathrm{~cm}$. L'ensemble est exposé à l'air libre dans l'actuel cimetière paroissial. Ce sarcophage est du même type qu'un exemplaire mis au jour lors de la fouille en 2013 du cimetière paroissial de Villenave-d'Ornon (Gironde). Deux autres furent découverts lors de la fouille du cimetière paroissial de Bruges (Gironde) en 2014, dont un était semblable à celui de Blanquefort et l'autre était différent par son décor soigné de stries régulières sur l'ensemble du couvercle ${ }^{5}$. Celui de Blanquefort, confectionné dans un calcaire local, jaune et à astéries, est légèrement plus grand que celui de Villenave-d'Ornon, un peu plus petit que celui décoré de Bruges et très proche de celui du même site dépourvu de décor. Cet exemple du site de Blanquefort complète ainsi le bilan régional élaboré récemment par Guillaume Rougé, Christian Scuiller et Yves Gleize ${ }^{6}$.

12 Hormis les indications fournies par les textes et cadastres évoqués précédemment, aucun autre indice ne permet de mieux appréhender l'emprise du cimetière et son évolution. Les textes indiquent que le cimetière de l'église Saint-Martin aurait accueilli des sépultures jusqu'en 1853, malgré un abandon officiel en 1809 pour cause d'insalubrité 7 . L'espace funéraire est transformé en place au cours du XIX siècle après le déplacement effectif du cimetière. 


\section{Apports de la fouille archéologique menée en 2014}

\section{La population inhumée}

Au total, dix-neuf sépultures d'individus en position primaire ont été observées, illustrant les phases médiévale et moderne de l'occupation funéraire autour de l'église, avec une même représentation en sujets adultes (9) et immatures (10).

En termes de stratigraphie, les sépultures modernes et contemporaines se situent entre l'unité stratigraphique 1005 (encaissant) et l'unité 1004 (abandon du cimetière). Les sépultures médiévales apparaissant dans deux unités stratigraphiques successives; la plus profonde, située ici directement sur le substrat, a livré à la fouille des tessons de céramique carolingienne. Ces niveaux d'inhumations médiévales ne peuvent être différenciés précisément, tous deux ayant livré des architectures funéraires similaires. Parmi les sépultures mises au jour, quatorze sont attribuables au second Moyen Âge, les plus anciennes ayant pu être datées des $\mathrm{XI}^{\mathrm{e}}$-XII ${ }^{\mathrm{e}}$ siècles (fig. 6).

Fig. 6 - Saint-Martin de Blanquefort : plan des vestiges mis au jour

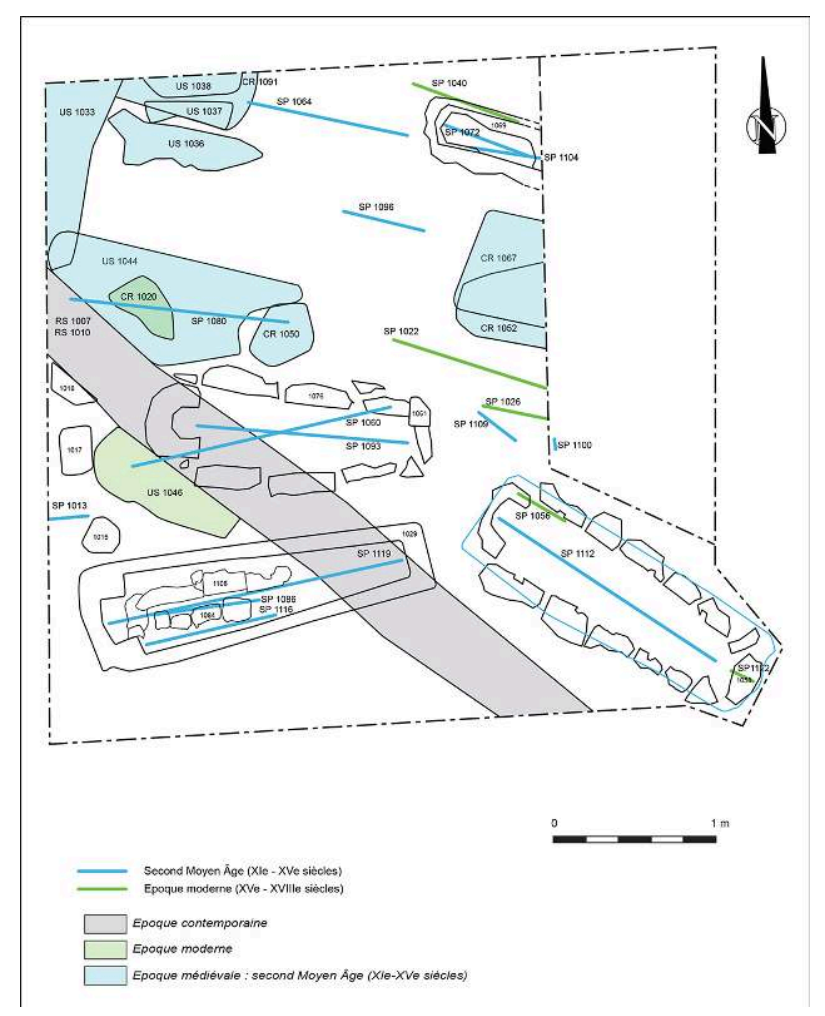

Les traits longs, bleus et verts, signalent les squelettes.

Topographie M.-P. Valleix, infographie J. Masson.

Parmi ces quatorze sujets, huit sont des individus adultes - un homme, une femme, six de sexe indéterminé - et six sont des individus immatures. L'échantillon est trop faible pour discuter du recrutement, toutefois nous pouvons remarquer que les classes d'âge les plus jeunes ( 0 et 1-4 ans) sont représentées tout comme celle des adolescents (10-14 ans). 

qui a été observé sur les sites voisins contemporains de Villenave-d'Ornon et de Bruges $^{8}$, traduisant des stress dans la vie de ces individus.

19 L'étude des assemblages céramiques issus de la fouille a mis en évidence un niveau qui se distingue des autres par son homogénéité chronologique et qui appartiendrait à la période carolingienne. C'est dans cette unité stratigraphique qu'ont été installées plusieurs sépultures, dont trois ont pu être datées des $\mathrm{XI}^{\mathrm{e}}$ et XII ${ }^{\mathrm{e}}$ siècles par une analyse radiocarbone. Les indices évoquant l'époque carolingienne restent trop peu nombreux et sur un espace trop restreint pour évoquer un habitat distinct d'un espace funéraire, contemporain ou pas, ou un cimetière habité, etc. S'il y a bien continuité d'occupation de cet espace, on ne peut affirmer, pour autant et dans l'état actuel des connaissances, une continuité dans la fonction funéraire du site.

Des monnaies datées des $\mathrm{XIV}^{\mathrm{e}}-\mathrm{XV}^{\mathrm{e}}$ siècles et des $\mathrm{XV}^{\mathrm{e}}-\mathrm{XVI}^{\mathrm{e}}$ siècles ont été prélevées dans le comblement d'une sépulture moderne et dans celui de fosses médiévales et modernes. Elles témoignent des remaniements fréquents au sein de l'espace funéraire, tout en s'accordant avec les datations des structures mises au jour.

\section{Différentes architectures funéraires}

21 Les architectures funéraires sont en pierre, en bois ou mixtes (pierre, bois, mortier) pour la période médiévale et en bois pour la période moderne. Le faible échantillon de Blanquefort ne permet pas de déceler une sélection typologique de ces architectures par âge ou par sexe.

Plusieurs coffrages en pierre (calcaire) ont été observés, mais le couvercle n'était pas conservé dans la majorité des cas et des sépultures postérieures étaient installées directement au-dessus. Une seule sépulture en coffrage en pierre était couverte par une dalle calcaire scellée à l'argile, aménageant un espace vide et clos (fig. 7). 
Fig. 7 - Sépulture en coffrage de pierre (calcaire blanc) aménagé avec une logette céphalique et couvert d'une dalle monolithe (calcaire jaune) scellé à l'argile orange

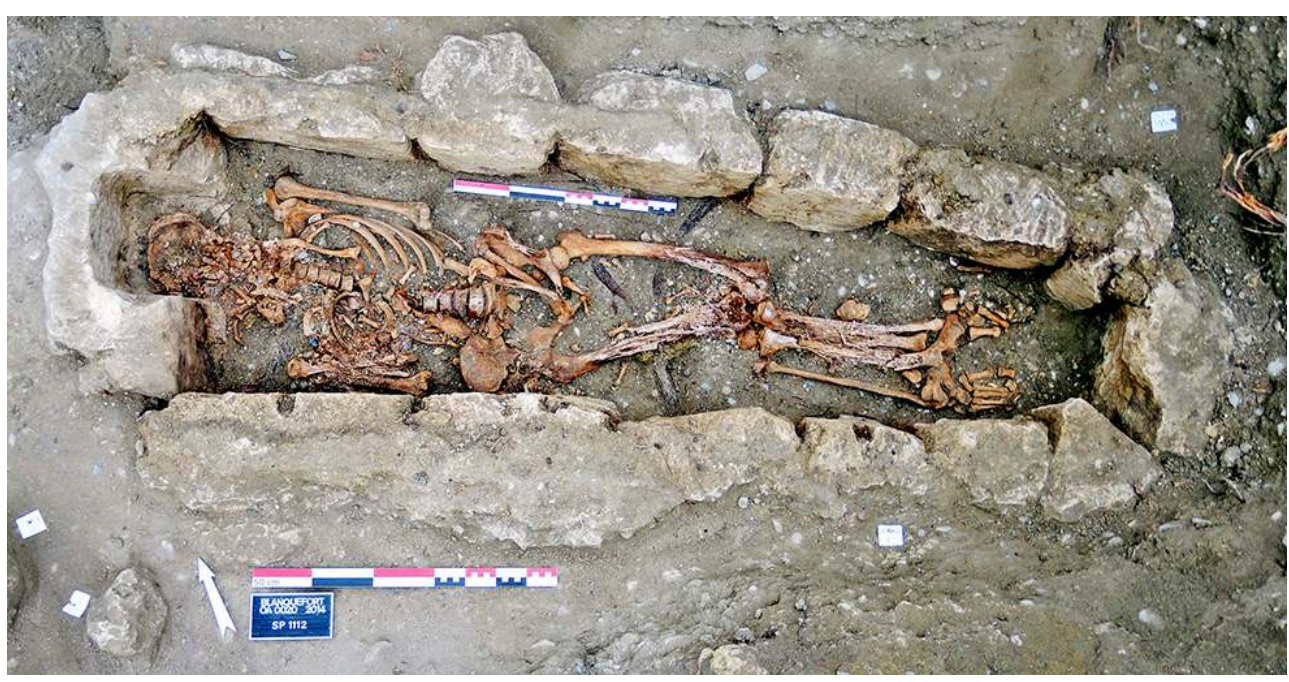

Cl. M. Rouzic

23 Un autre coffrage en pierre, destiné à un sujet immature, était en grande partie recouvert de mortier, où des négatifs de planches en bois étaient visibles : des éléments en bois semblent avoir été utilisés pour servir de couvercle ou installés pour soutenir un couvercle d'une autre nature, non retrouvé en fouille (fig. 8).

Fig. 8 - Sépulture en coffrage de pierre recouvert de mortier de chaux conservant des traces d'éléments en bois

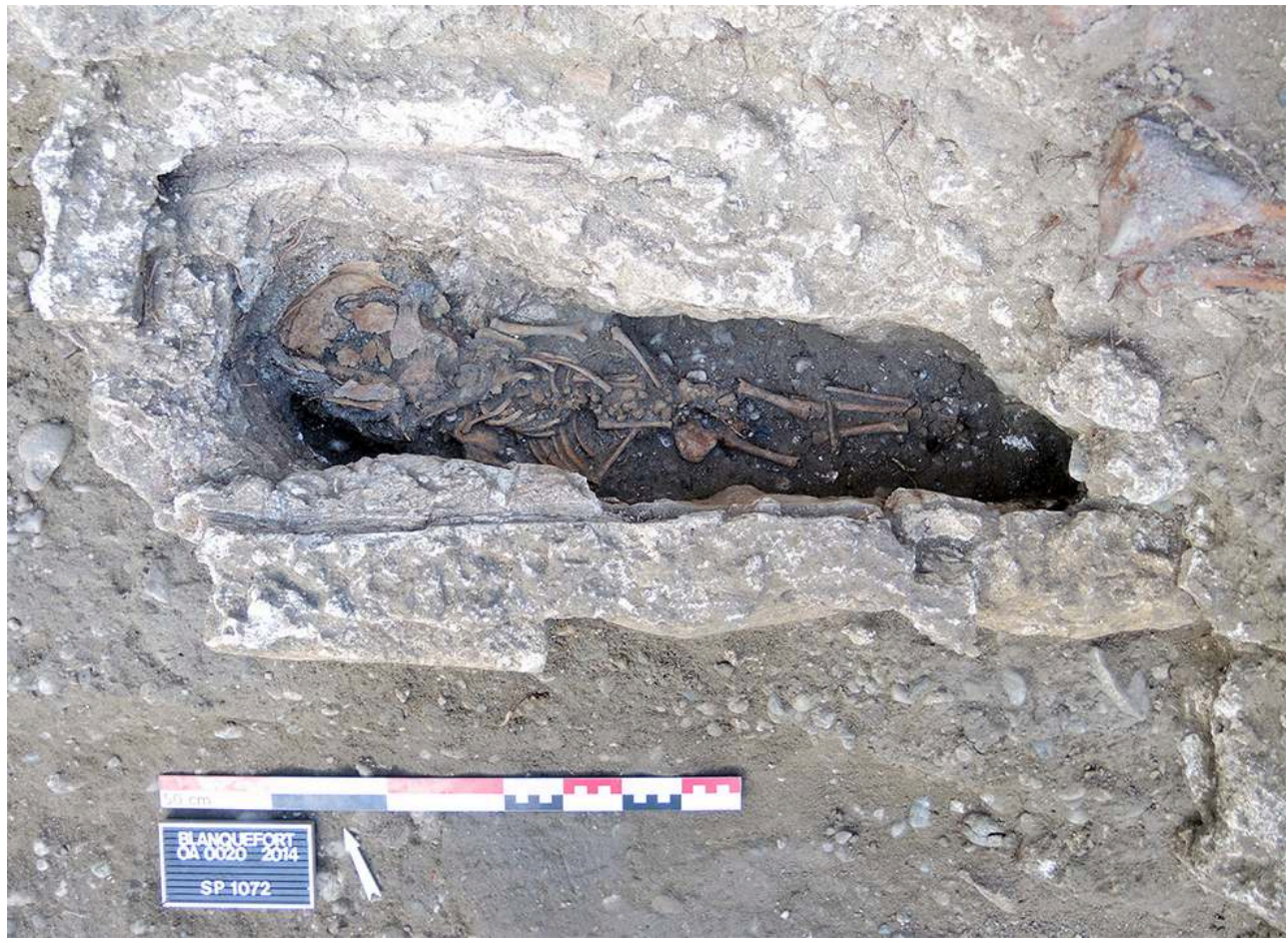

Cl. M. Hautin

Une cuve de sarcophage en calcaire avec logette céphalique trapézoïdale a également été mise au jour (fig. 9), avec trois périodes successives d'utilisation. 
Fig. 9 - Sépulture en sarcophage de plan trapézoïdal avec une logette céphalique en queue d'aronde

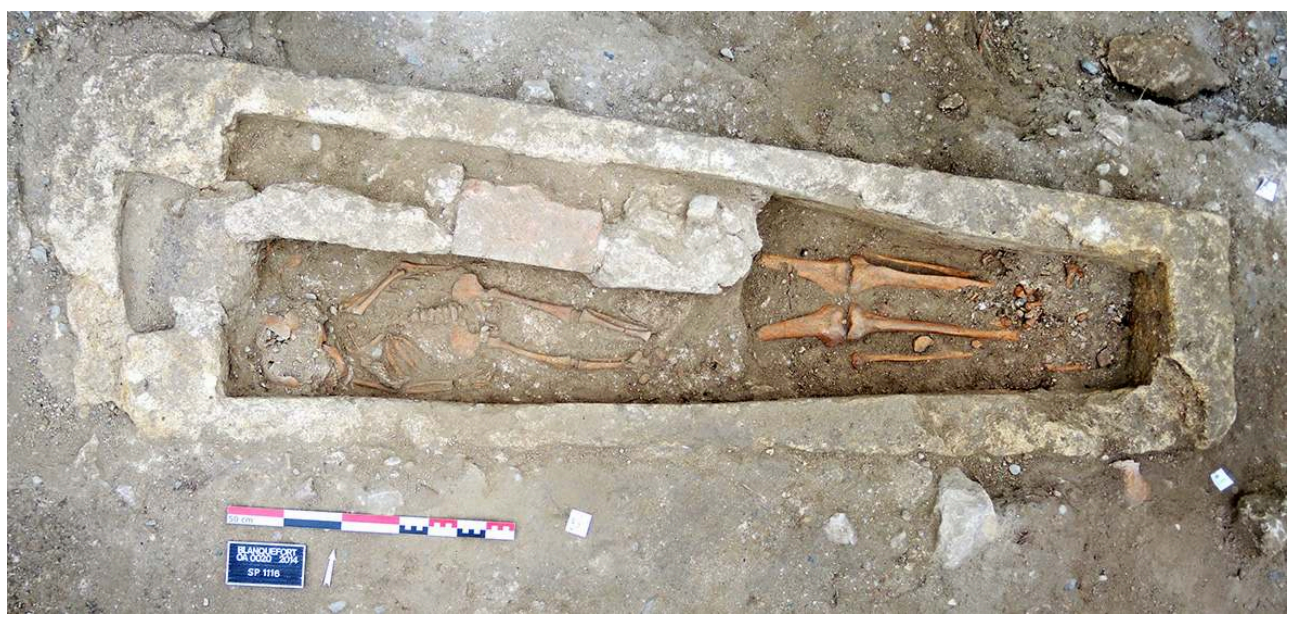

Ce sarcophage a été rouvert pour y aménager un coffrage et inhumer un sujet immature.

Cl. M. Rouzic

Un premier sujet adulte y avait été déposé, puis cette sépulture avait été ouverte à nouveau pour y aménager un coffrage en pierre partant de la logette céphalique et réduisant l'espace intérieur de moitié, pour y installer un sujet immature, le tout étant refermé par le couvercle initial du sarcophage. Celui-ci a ensuite été recreusé de manière à abriter la fosse d'un troisième sujet, immature également (fig. 10). 
Fig. 10 - Sujet immature (1116) inhumé dans le sarcophage d'un premier défunt adulte (1119) et sépulture d'un sujet immature (1086) aménagée sur le couvercle du sarcophage

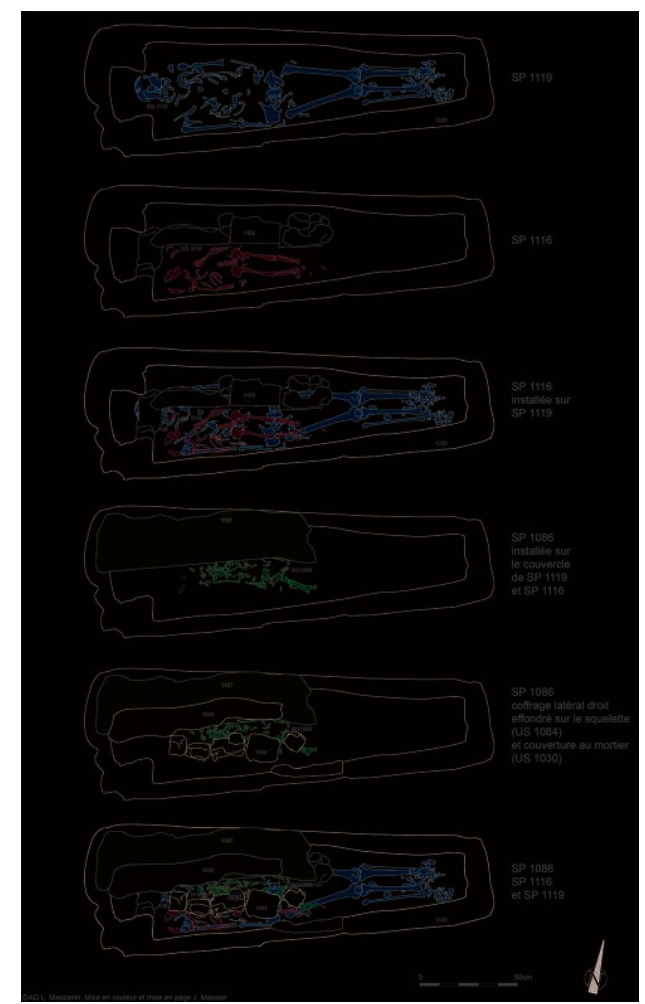

L'usage du mortier a été observé pour le fond et le coffrage du sujet immature présent dans le sarcophage, de même pour le sujet immature installé sur le couvercle, qui, lui, a en outre reçu une couverture de mortier - éléments en bois à envisager, mais non perçus à la fouille.

Infographie L. Maccanin, mises en couleur et en page J. Masson.

Le mortier de chaux était fréquemment utilisé dans l'aménagement des sépultures, en fond de fosse et dans certains cas aussi pour former des bords, voire une couverture, probablement en association avec des éléments en bois, qui n'ont pas pu être reconnus à la fouille. Enfin, des contenants en bois ont été observés dans les niveaux attribués à l'occupation moderne.

\section{Conclusion}

Les résultats issus de la fouille sur le cimetière de Saint-Martin de Blanquefort révèlent le potentiel de ce type d'intervention de petite envergure. Malgré une fenêtre d'observation assez réduite, ils sont tout à fait représentatifs des différentes problématiques développées dans la recherche la plus actuelle en archéologie funéraire médiévale. Ils seront intégrés dans une étude de synthèse, regroupant les résultats des opérations menées sur les cimetières paroissiaux des communes du même territoire métropolitain - Villenave-d'Ornon, Bruges, Gradignan et Mérignac -, afin d'avancer sur les questions liées à l'implantation des lieux de culte, à la continuité de l'occupation funéraire attenante, à la typologie de l'architecture funéraire, tout en prenant soin de toujours lier, quand le site s'y prête, étude de l'espace funéraire et analyse de l'édifice associé.

Reçu : 10 août 2017 - Accepté : 27 novembre 2017 


\section{NOTES}

1. C. DOULAN avec la collaboration de X. CHARPENTIER, Carte archéologique de la Gaule, t.33/2 (Bordeaux), Paris, 2013.

2. V. ELIZAGOYEN, Rapport de diagnostic, Blanquefort place de l'église (Gironde), rapport final d'opération de fouille, Inrap/ministère de la Culture/SRA Aquitaine, Bordeaux, 2013.

3. Archives départementales de la Gironde, G 652, fol. 66-75.

4. V. ELIZAGOYEN, Rapport de diagnostic..., op. cit. et $\mathrm{H}$. BRET, La vie religieuse à Blanquefort au $\mathrm{XX}^{e}$ siècle, Blanquefort, 2006.

5. H. RÉVEILLAS, Villenave-d'Ornon, place de l'église Saint-Martin, rapport final d'opération de fouille, Service d'archéologie préventive de Bordeaux métropole/ministère de la Culture/SRA Aquitaine, Bordeaux, 2014, US 2255 ; J. MAsson, Place de l'église Saint-Pierre à Bruges (Gironde), Rapport final d'opération de fouille, Bordeaux, Centre d'archéologie préventive de Bordeaux métropole/ministère de la Culture/SRA Aquitaine-Limousin-Poitou-Charentes, Bordeaux, 2017, US 1732 et US 1756.

6. G. ROUGÉ, C. SCUILLER et Y. GLEIZE, "Cartographie des sites à sarcophages en Aquitaine ", in I. CARTRON, F. HENRION et C. SCUILLER (éd.), Les sarcophages de l'Antiquité tardive et du haut Moyen Âge : fabrication, utilisation, diffusion (éd. Aquitania, suppl. 34), Bordeaux, 2015, p. 149-154.

7. G. DABADIE, Blanquefort et sa région à travers les siècles, Bordeaux, 1952, p. 117.

8. H. RÉVEILLAS, Villenave-d'Ornon..., op. cit. ; J. MASSON, Place de l'église Saint-Pierre à Bruges..., op. cit.

\section{INDEX}

Mots-clés : haut Moyen Âge, Moyen Âge central, église paroissiale, cimetière, sépulture, sarcophage, coffrage

\section{AUTEURS}

\section{JULIETTE MASSON}

Archéologue responsable d'opérations Moyen Âge-époque moderne, Centre d'archéologie préventive de Bordeaux métropole/UMR 5607-AUSONIUS université Bordeaux Montaigne

\section{HÉLÈNE RÉVEILLAS}

Archéo-anthropologue responsable d'opérations, Centre d'archéologie préventive de Bordeaux métropole/UMR 5199-PACEA université de Bordeaux

\section{MIKAËL ROUZIC}

Archéo-anthropologue contractuel, Centre d'archéologie préventive de Bordeaux métropole/ UMR 5199-PACEA université de Bordeaux 